\title{
论 文
}

\section{大气环境的硫同位素组成及示踪研究}

\author{
张苗云 ${ }^{(1)}$ ，王世杰 ${ }^{(1)}$ ，马国强 ${ }^{(2)}$, 周怀中 ${ }^{(2)}$, 傅军 ${ }^{3}$ \\ (1) 中国科学院地球化学研究所环境地球化学国家重点实验室, 贵阳 550002; \\ (2) 金华市环境监测中心站, 金华 321013; \\ (3) 浙江省环境监测中心, 杭州 310012 \\ * 联系人, E-mail: wangshijie@ vip.gyig.ac.cn
}

收稿日期: 2009-08-19; 接受日期: 2010-05-25

国家重点基础研究发展计划(编号：2006CB403200)、国家自然科学基金创新群体项目(批准号：40721002)、浙江省科技计划项目(编号： 2004C33072)、浙江省环保科研计划项目(编号: 200720)和金华市科技计划项目(编号: 2008-3-001)资助

\begin{abstract}
摘要大气 $\mathrm{SO}_{2}$ 和气溶胶硫酸盐是导致大气环境酸化, 加剧酸沉降污染的重要因素. 由于稳 定硫同位素组成的“指纹”特征, 硫同位素示踪技术已被广泛地应用到环境领域中的硫循环和硫 源解析等研究. 本文对浙江中部地区金华市的大气环境进行了连续的观测, 并分析测定了大气 $\mathrm{SO}_{2}$ 和气溶胶硫酸盐的硫同位素组成. 结果表明, 研究区域大气 $\mathrm{SO}_{2}$ 的 $\delta^{34} \mathrm{~S}$ 值变化范围是 $1.0 \%$ 7.5\%o, 年均值为 $4.7 \% 0 \pm 2.3 \%$, 气溶胶 $\delta^{34} \mathrm{~S}$ 值变化范围是 $6.4 \%$ $9.8 \%$, 年均值为 $8.1 \% 0 \pm 1.0 \%$ 。 . 大气 $\mathrm{SO}_{2}$ 的 $\delta^{34} \mathrm{~S}$ 值存在明显的季节性变化特征, 冬季为 $7.0 \%$, 夏季为 $3.3 \%$, 研究 指出单一的控制因素并不能完全解释研究区域大气 $\mathrm{SO}_{2}$ 硫同位素的季节性变化, 认为同位素平 衡分馏的温度效应和夏季富轻同位素的生物成因硫的大量释放可能是引起这种季节性变化的 主要控制因素. 另外, 文中还讨论了大气 $\mathrm{SO}_{2}$ 氧化形成硫酸盐的 $\delta^{34} \mathrm{~S}$ 模式, 认为在浙江中部地 区大气 $\mathrm{SO}_{2}$ 氧化反应中多相氧化处于主导地位, 同时大气环境的相对湿度对大气 $\mathrm{SO}_{2}$ 的氧化机 制有着重要的影响.
\end{abstract}

关键词

硫同位素

$\mathrm{SO}_{2}$

气溶胶

均相氧化

多相氧化;
大气环境中的硫来自天然源释放和人为活动排 放, 是造成酸雨污染的主要空气污染物. $\mathrm{SO}_{2}$ 是人为 排放的主要硫化合物, 也是大气环境中的主要致酸 前体物. 大气 $\mathrm{SO}_{2}$ 等低氧化态的硫化合物化学性质相 对活泼, 在大气中很快就被氧化, 最终通过气相的均 相氧化或液相的多相氧化形成硫酸盐 ${ }^{[1,2]}$. 硫酸盐是 大气中主要的气溶胶粒子之一, 也是大气中云凝结 核的主要成分 ${ }^{[3,4]}$. 硫酸盐在对流层和低平流层化学 中有着十分重要的作用, 它能够在大气粒子表面沉 淀或者通过气一粒转化过程形成气溶胶粒子 ${ }^{[5,6]}$. 对
流层的硫酸盐气溶胶引起酸雨污染, 通过直接和间 接辐射强迫影响全球气候, 同时还对人类的呼吸系 统产生威胁 ${ }^{[7 ~ 10]}$. 因此, 确定不同地区大气硫来源的 相对组成及其在源、汇间的迁移转化对定量评估区域 环境硫酸盐气溶胶的物理和化学效应, 了解大气 $\mathrm{SO}_{2}$ 和硫酸盐气溶胶对全球环境的影响具有十分重要的 意义.

金华市位于浙江省中部地区，钱塘江上游，金䓢 盆地东缘, 多丘陵盆地, 地势南北高、中部低, 属典 型的亚热带季风气候，四季分明，湿润多雨. 本文通

英文引用格式: Zhang M Y, Wang S J, Ma G Q, et al. Sulfur isotopic composition and source identification of atmospheric environment in central Zhejiang, China. Sci China Earth Sci, 2010, 53: 1717-1725, doi: 10.1007/s11430-010-4017-9 
过对金华市区 2004 年到 2005 年大气污染状况的实时 监测及大气 $\mathrm{SO}_{2}$ 和气溶胶硫酸盐硫同位素的分析测 定, 以了解研究区域大气 $\mathrm{SO}_{2}$ 和气溶胶硫酸盐硫同位 素组成及变化规律, 认识大气环境中 $\mathrm{SO}_{2}$ 形成硫酸盐 的氧化途径, 最终达到解析大气硫的不同来源的目 的.

\section{1 实验部分}

\section{1 样品采集和处理}

大气 $\mathrm{SO}_{2}$ 和气溶胶样品通过 $\mathrm{TH} 1000 \mathrm{C}$ 型智能大 容量空气总悬浮微粒采样器采集, 用经过前处理的 K-49 型超细玻璃纤维滤膜 $(20 \mathrm{~cm} \times 25 \mathrm{~cm})$ 分别采集大 气 $\mathrm{SO}_{2}$ 和气溶胶样品. 采集大气 $\mathrm{SO}_{2}$ 的玻璃纤维滤膜 要先经过 $2 \% \mathrm{~K}_{2} \mathrm{CO}_{3}+2 \%$ 甘油溶液浸泡处理, 然后 $50^{\circ} \mathrm{C}$ 烘干. 采集气溶胶的玻璃纤维滤膜要先放入烘 箱 $90^{\circ} \mathrm{C}$ 烘 $6 \mathrm{~h}$, 然后用二次蒸馏水浸泡 $4 \mathrm{~h}, 50^{\circ} \mathrm{C}$ 烘干, 再用 $1: 1$ 的磷酸浸泡 $30 \mathrm{~min}$, 最后用去离子水反复冲 洗, 直至滤膜显中性. 采样时分别将准备好的滤膜装 在双层的采样架上, 上层采集气溶胶, 下层采集大气 $\mathrm{SO}_{2}$, 采样流量 $100 \mathrm{~L} / \mathrm{min}$, 采样时间 $24 \mathrm{~h}$ 为一个大气 $\mathrm{SO}_{2}$ 和气溶胶样品 ${ }^{[11]}$.

采集后的气溶胶样品按下面过程提取硫酸盐, 剪取 $1 / 4$ 采样滤膜, 剪碎后放入 $50 \mathrm{~mL}$ 烧杯中, 加入 $20 \mathrm{~mL}$ 去离子水, 搅拌均匀后, 再用 $0.45 \mu \mathrm{m}$ 有机微 孔滤膜过滤, 在滤液中加入 $1 \mathrm{~mL} 2 \mathrm{~mol} / \mathrm{L}$ 的 $\mathrm{BaCl}_{2}$ 溶 液, 出现 $\mathrm{BaSO}_{4}$ 白色沉淀, 用离心的方法分离 $\mathrm{BaSO}_{4}$ 沉淀, 再用去离子水清洗 $\mathrm{BaSO}_{4}$ 沉淀数次, 最后 $50^{\circ} \mathrm{C}$ 烘干得到纯净的硫酸钡. 大气 $\mathrm{SO}_{2}$ 样品的硫酸盐提取 过程需要加入 $\mathrm{H}_{2} \mathrm{O}_{2}$ 先将亚硫酸盐氧化成硫酸盐, 其他 步骤与气溶胶样品的硫酸盐提取的过程基本相同 ${ }^{[12]}$.

\section{2 分析测试}

样品硫同位素分析在中国科学院地球化学研究 所环境地球化学国家重点实验室的 IsoPrime 连续流 质谱仪上分析测定. 硫同位素分析采用的工作标准 有 VCDT 标准 IAEA-S- $1\left(\mathrm{Ag}_{2} \mathrm{~S},-0.30 \%\right.$ ) 、美国国家 标准和技术研究所标准 NBS-127(重晶石, 20.3\% )和 国家参考标准 GBW04415 $\left(\mathrm{Ag}_{2} \mathrm{~S}, 22.15 \%\right.$ o), 分析结果 相对于国际标准 $\mathrm{CDT}$, 以 $\delta^{34} \mathrm{~S}$ 值表示, 分析测试的标 准偏差为 $\pm 0.2 \% o(n=10)$.

\section{2 结果与讨论}

\section{1 大气中 $\mathrm{SO}_{2}$ 和 $\mathrm{NO}_{2}$ 的浓度水平}

通过对研究区域 2004 2005 年空气质量自动监 测系统 $\mathrm{SO}_{2}$ 和 $\mathrm{NO}_{2}$ 监测数据的统计分析, 结果表明研 究区域环境空气中 $\mathrm{SO}_{2}$ 日均值浓度在 $0.004 ~ 0.204$ $\mathrm{mg} / \mathrm{m}^{3}$ 之间, 2004 和 2005 年的年均值分别为 0.044 和 $0.048 \mathrm{mg} / \mathrm{m}^{3} ; \mathrm{NO}_{2}$ 日均值浓度在 $0.010 \sim 0.135 \mathrm{mg} / \mathrm{m}^{3}$ 之间, 2004 和 2005 年的年均值分别为 0.057 和 0.053 $\mathrm{mg} / \mathrm{m}^{3}$.

图 1 分别反映了 2004 2005 年研究区域大气 $\mathrm{SO}_{2}$, $\mathrm{NO}_{2}$ 浓度的时间变化. 两种污染成分都表现出比较明 显的季节性变化, $\mathrm{SO}_{2}$ 浓度冬、春季比较高, 夏、秋季 相对较低, 其中 12 月 $\mathrm{SO}_{2}$ 浓度最高, 而在 6, 7 月达到 最低. $\mathrm{NO}_{2}$ 浓度变化各个季节之间不是分得非常清晰, 但每年 10 月以后的浓度都逐渐升高, 这种相对较高 的浓度水平一直维持到 4 月底, 5 月份开始逐渐降低, 6 月底到 8 月初达到最低, 表现出明显的时间变化序 列.

\section{2 大气 $\mathrm{SO}_{2}$ 和气溶胶硫同位素组成特征}

本研究在 2005 年对研究区域大气 $\mathrm{SO}_{2}$ 和气溶胶 进行了为期一年的硫同位素观测, 每月中旬分别采 集一个大气 $\mathrm{SO}_{2}$ 和气溶胶样品, 通过前处理提取硫酸 盐, 分析测定样品的硫同位素组成, 表 1 是 2005 年大 气 $\mathrm{SO}_{2}$ 和气溶胶的硫同位素分析测定结果.

表中数据表明, 浙江中部地区大气 $\mathrm{SO}_{2}$ 的 $\delta^{34} \mathrm{~S}$ 值 变化范围在 $1.0 \%$ 7.5\%o之间, 年均值为 $4.7 \%$ 叛 $2.3 \%$, 而气溶胶 $\delta^{34} \mathrm{~S}$ 值变化范围在 $6.4 \%$ 9.8\% 之间, 年均

表 1 大气 $\mathrm{SO}_{2}$ 和气溶胶的硫同位素组成 $\left(\delta^{84} \mathrm{~S} \%\right)$

\begin{tabular}{ccc}
\hline 时间 & 大气 $\mathrm{SO}_{2}$ & 气溶胶 \\
\hline 2005 年 1 月 & 7.5 & 8.8 \\
2005 年 2 月 & 7.3 & 7.5 \\
2005 年 3 月 & 6.0 & 7.5 \\
2005 年 4 月 & 7.5 & 8.9 \\
2005 年 5 月 & 1.0 & 9.8 \\
2005 年 6 月 & 3.7 & 8.3 \\
2005 年 7 月 & 1.5 & 6.8 \\
2005 年 8 月 & 4.6 & 8.1 \\
2005 年 9 月 & 2.8 & 9.0 \\
2005 年 10 月 & 5.1 & 8.3 \\
2005 年 11 月 & 3.7 & 6.4 \\
2005 年 12 月 & 6.2 & 7.6 \\
\hline
\end{tabular}



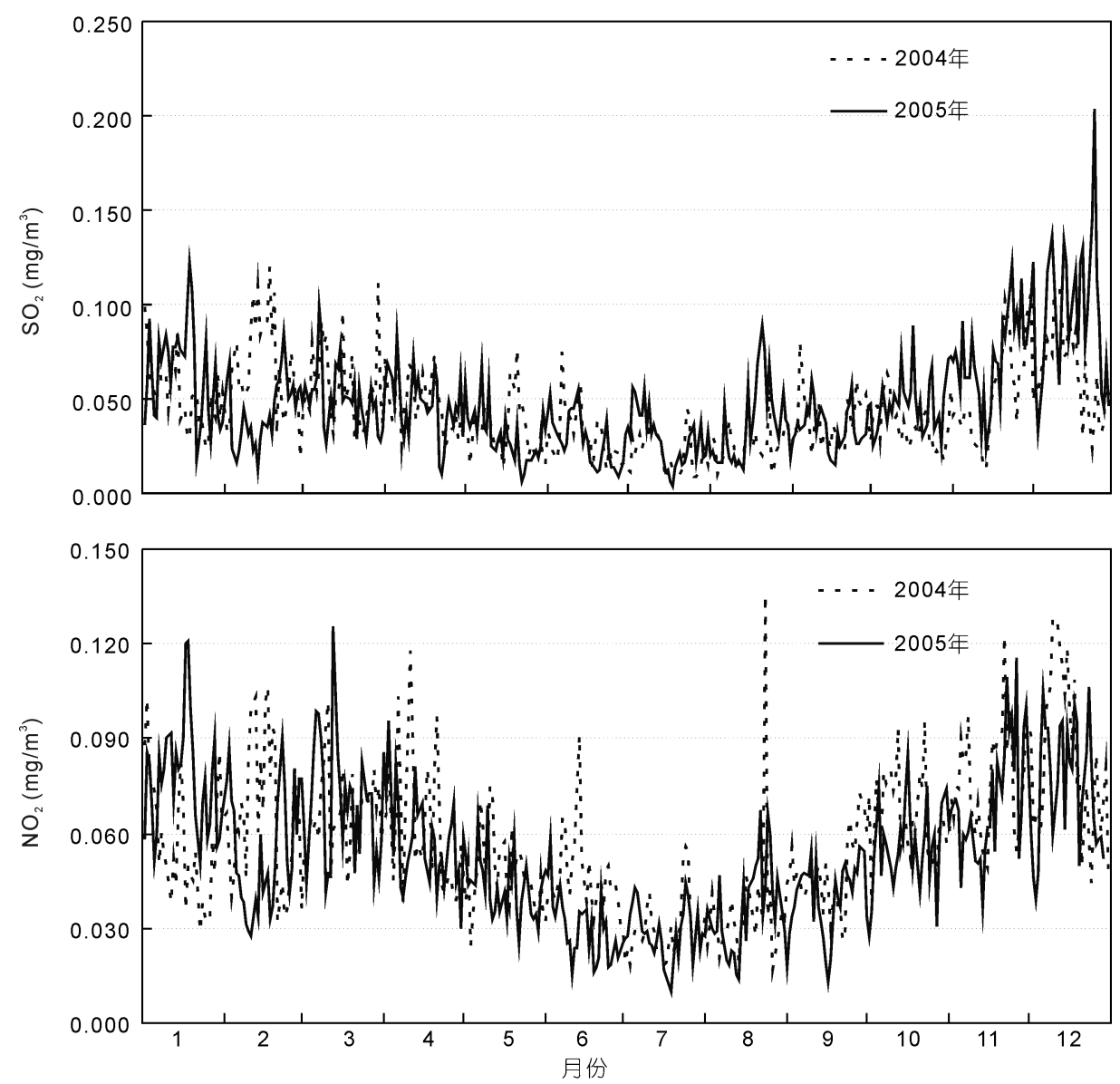

图 $12004 \sim 2005$ 年大气 $\mathrm{SO}_{2}$ 和 $\mathrm{NO}_{2}$ 浓度的时间变化

值为 $8.1 \% 0 \pm 1.0 \%$. 气溶胶 $\delta^{34} \mathrm{~S}$ 值毫无例外都大于相 应的大气 $\mathrm{SO}_{2}$ 的 $\delta^{34} \mathrm{~S}$ 值, 两者的年均值相差 3.4\%o, 这 和国内外观测到的大气硫同位素组成一致 ${ }^{[12 \sim 17]}$. 这 种现象也符合燃煤硫同位素分馏规律, 即燃煤释放 出的 $\mathrm{SO}_{2}$ 总是比原煤相对富集轻硫同位素, 而释放出 的颗粒物总是比原煤相对富集重硫同位素 ${ }^{[18,19]}$. 从 时间上来看, 研究区域大气 $\mathrm{SO}_{2}$ 的 $\delta^{34} \mathrm{~S}$ 值季节性变化 明显, 冬季到春季的 4 月份 $\delta^{34} \mathrm{~S}$ 值最高, 春末到夏季 的 $\delta^{34} \mathrm{~S}$ 值最低, 秋季比夏季稍高(图 2). 研究区域大 气降水硫同位素 $\delta^{34} \mathrm{~S}$ 值变化范围在 $0.5 \%$ 14.2\% 之间, 平均值约为 $5.0 \%$, 同时 $\delta^{34} \mathrm{~S}$ 值也存在冬季高夏季低 的季节波动现象 ${ }^{[11]}$, 这与大气 $\mathrm{SO}_{2}$ 硫同位素组成的 季节变化特征是一致的. 另一方面, 大气 $\mathrm{SO}_{2}$ 年均 $\delta^{34} \mathrm{~S}$ 值比气溶胶年均 $\delta^{34} \mathrm{~S}$ 值更接近大气降水年均 $\delta^{34} \mathrm{~S}$ 值, 这些结果说明大气 $\mathrm{SO}_{2}$ 对降水硫同位素组成的影 响更明显, 也就是说降水硫同位素的组成中大气 $\mathrm{SO}_{2}$ 的相对贡献可能要大于气溶胶. 气溶胶 $\delta^{34} \mathrm{~S}$ 值全年的
变化幅度不大, 基本围绕在年均值附近上下波动, 春 季的 $\delta^{34} \mathrm{~S}$ 值相对稍高, 冬、夏和秋季的 $\delta^{34} \mathrm{~S}$ 值则几乎 完全一致(图 2).

国内对大气 $\mathrm{SO}_{2}$ 和气溶胶的硫同位素组成已做 了一定的研究工作. 洪业汤等 ${ }^{[20]}$ 、刘广深等 ${ }^{[21]}$ 和张鸿 斌等 ${ }^{[16,17]}$ 分别对贵阳地区和华南的部分地区大气 $\mathrm{SO}_{2}$ 和气溶胶的硫同位素进行了分析测定, 图 3 是不 同地区大气 $\mathrm{SO}_{2}$ 和气溶胶硫同位素组成的变化范围. 浙江中部地区与贵阳地区和湘桂走廊地区有着明显 的区别，与华南的珠江三角洲地区及衡阳地区也有 一定的差别, 这种区域分异现象主要与各地区使用 的化石燃料的不同来源密切相关, 也是造成大气 $\mathrm{SO}_{2}$ 和气溶胶的硫同位素组成区域分异的根本原因. 不 同地区的化石燃料由于其形成的地质背景不同，它 们的硫同位素组成有很大的差异. 浙江中部地区消 耗的煤炭主要来自中国北方地区，与贵阳地区和湘 桂走廊地区差异明显. 图 4 给出了中国不同地区煤炭 


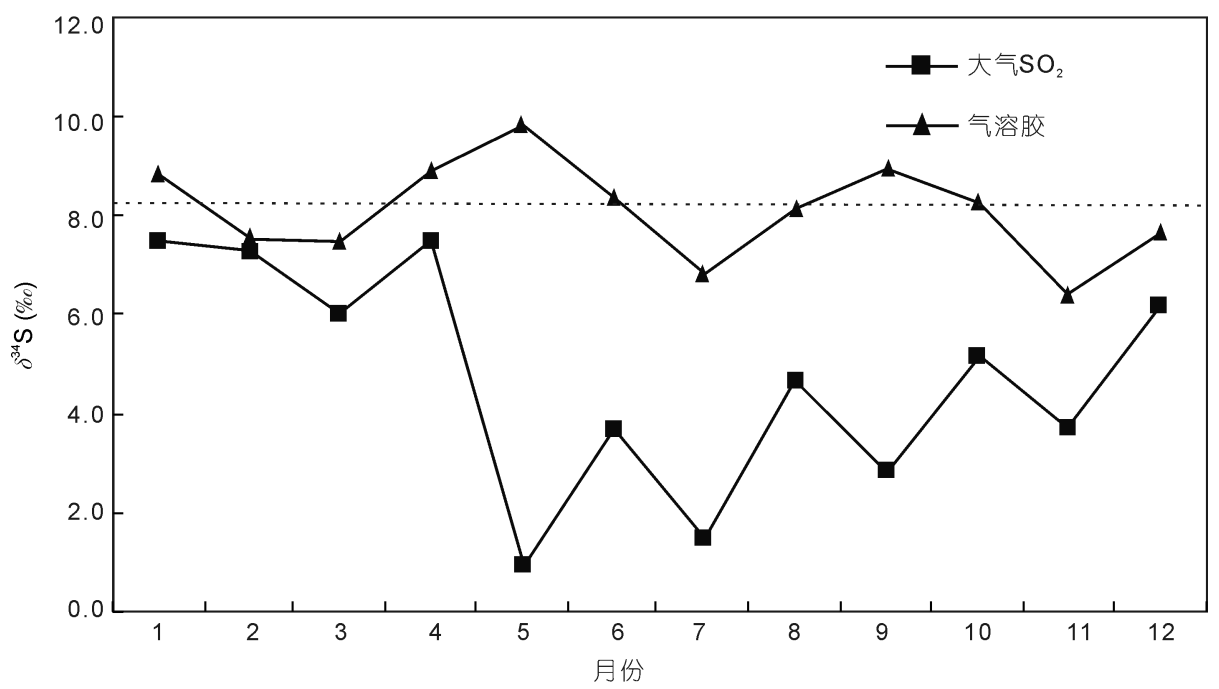

图 2 大气 $\mathrm{SO}_{2}$ 和气溶胶 $\delta^{34} \mathrm{~S}$ 值的时间变化

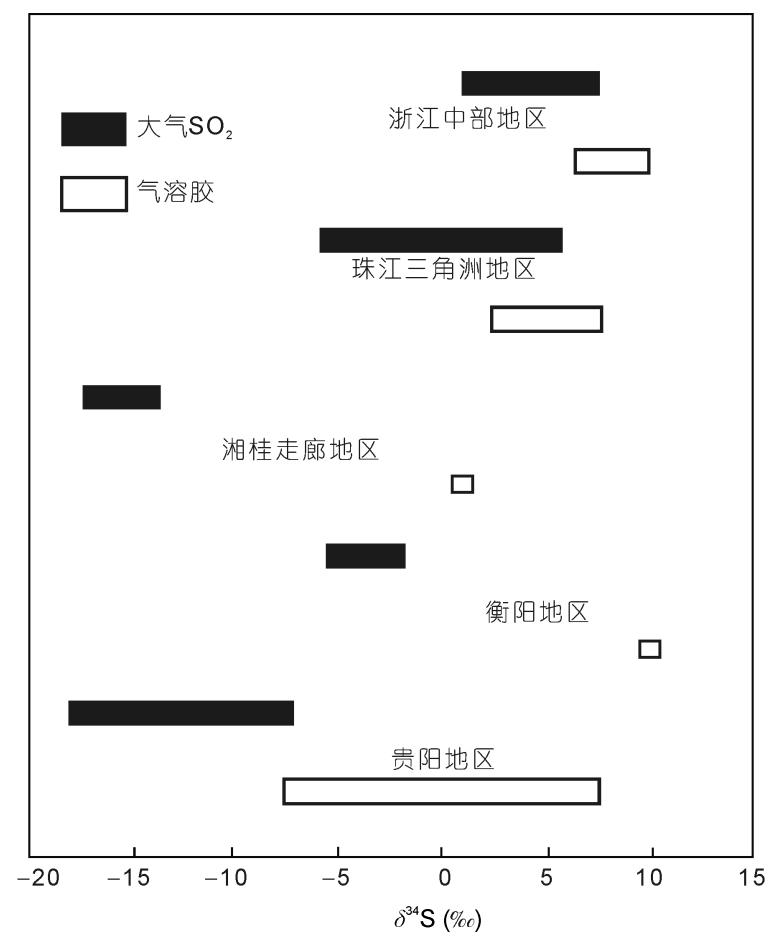

图 3 不同地区大气 $\mathrm{SO}_{2}$ 和气溶胶硫同位素组成

的硫同位素组成分布状况, 从图中可以看出, 煤因产 地不同硫同位素组成变化较大, 煤炭硫同位素组成 的区域特征造成其燃烧产物一排放的污染物 $\left(\mathrm{SO}_{2}\right.$ 和 颗粒物)硫同位素组成的不同 ${ }^{[18]}$. 中日合作开展对中 国贵阳、北京、大连、长春、哈尔滨、上海、南京、 青海瓦里关、日本 Tsukuba 等城市的大气 $\mathrm{SO}_{2}$ 和气溶 胶硫同位素及铅同位素组成研究 ${ }^{[12]}$, 同样观察到了
这种明显的区域分异现象，北方地区大连、长春、哈 尔滨的大气 $\mathrm{SO}_{2}$ 的 $\delta^{34} \mathrm{~S}$ 值大约为 $5 \%$ ，而南方的贵阳 约在 $-3 \%$ o左右, 南京和上海的均值则在 $3 \%$ o左右. 他 们认为大气 $\mathrm{SO}_{2}$ 硫同位素组成的区域分异与各地区 煤的硫同位素组成相一致, 是由化石燃料的硫同位 素组成差异造成的 ${ }^{[12]}$.

浙江中部地区大气 $\mathrm{SO}_{2}$ 和气溶胶硫同位素组成 大体上与上海和南京的大气 $\mathrm{SO}_{2}$ 和气溶胶硫同位素 组成相似, 大气 $\mathrm{SO}_{2}$ 和气溶胶 $\delta^{34} \mathrm{~S}$ 值全部落在 $0 \% 0 \sim 10 \%$ 之间. 从季节变化来看, 上海气溶胶 $\delta^{34} \mathrm{~S}$ 值 冬季为 $6.3 \%$, 夏季为 $5.4 \%$, 两者相差很小, 与浙江 中部地区一样, 季节变化都不明显, 但浙江中部地区 气溶胶硫同位素的均值要稍高于上海. 大气 $\mathrm{SO}_{2}$ 硫同 位素组成各地都表现出一定的季节变化, 大约在 1\%o 3\%o之间，其中上海的季节变化趋势与其他地区 相反 ${ }^{[12]}$. Norman 等 ${ }^{[22]}$ 和 Novak 等 ${ }^{[15]}$ 分别在加拿大和 欧洲中部的捷克也都观察到了明显的季节变化特征. Norman 等 ${ }^{[22]}$ 认为加拿大北极 Alert 地区的季节性变 化主要是由于不同季节硫的来源不同，并且这些硫 源具有各自独特硫同位素组成特征，但 Alert 地区硫 同位素组成季节变化趋势是夏季高冬季低，恰好与 上海的季节变化一致. Novak 等 ${ }^{[15]}$ 同样观测到欧洲中 部的捷克大气 $\mathrm{SO}_{2}$ 硫同位素组成存在夏季高冬季低 的季节变化趋势, 并且认为欧洲中部捷克冬季低的 硫同位素组成是由于受到当地低 $\delta^{34} \mathrm{~S}$ 值的燃煤排放 的影响, 当地煤以富轻硫同位素为特征, 平均 $\delta^{34} \mathrm{~S}$ 值 约为 $1.6 \%$. 总的来说, 大气环境硫同位素季节性变 


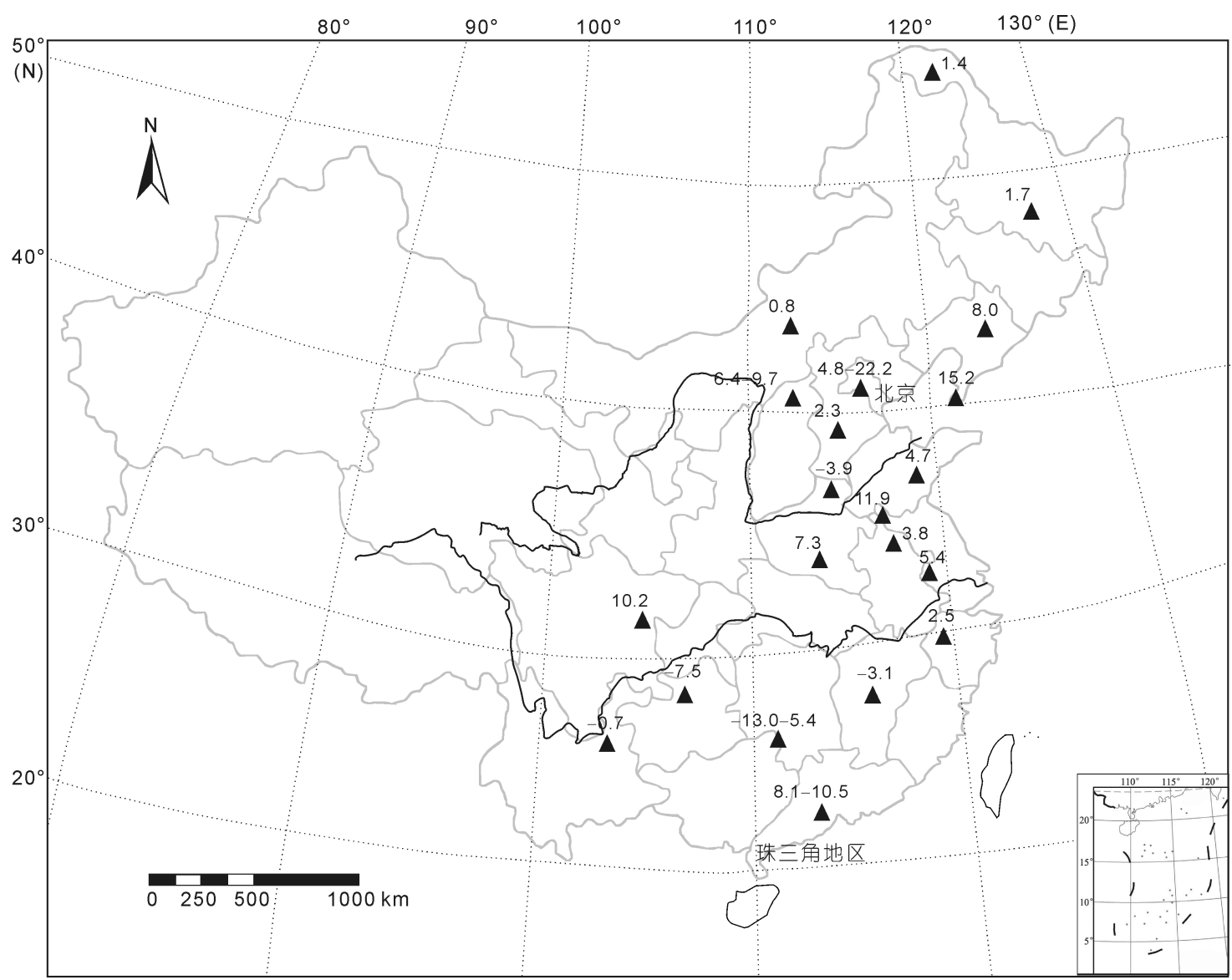

图 4 中国不同地区煤炭硫同位素组成分布图 $\left(\delta^{34} \mathrm{~S} \%\right.$ )

图中数据引自参考文献 $[17,18]$

化的控制因素主要有两种解释, 一些学者强调富轻 同位素特征的生物成因硫的作用，由于它们在夏季 的大量释放降低了大气中硫同位素的组成 ${ }^{[23 ~ 26]}$, 从 而形成冬季富重硫同位素、夏季富轻硫同位素的季节 变化特征. 还有一些学者则认为可以用硫酸盐离子 形成过程中 $\mathrm{HSO}_{3}{ }^{-}-\mathrm{SO}_{2}$ 反应体系同位素平衡分馏引 起的温度效应来解释, 而不是不同硫释放源的硫同 位素变化造成的 ${ }^{15,27,28]}$. 他们指出 $\mathrm{HSO}_{3}{ }^{-}-\mathrm{SO}_{2}$ 反应体 系硫的同位素分馏系数从 $25^{\circ} \mathrm{C}$ 的 1.0173 减小到 $70^{\circ} \mathrm{C}$ 的 1.0107 , 相当于下降 $0.145 \% \sigma^{\circ} \mathrm{C}^{[29]}$. 另外, 理论计 算表明 $\mathrm{SO}_{3}{ }^{2-}-\mathrm{SO}_{2}$ 反应体系的硫同位素也存在温度效 应，随着温度升高硫同位素下降 $0.08 \% d /{ }^{\circ} \mathrm{C}^{[30,31]}$. 按照 落在 $0.08 \sim 0.145 \%{ }^{\circ} \mathrm{C}$ 之间的 $0.10 \%{ }^{\circ} \mathrm{C}$ 的温度梯度系 数估算, 同位素平衡分馏的温度效应能够引起大约 $2 \%$ 3 3\%o的季节性变化. 浙江中部地区大气 $\mathrm{SO}_{2}$ 冬季 的 $\delta^{34} \mathrm{~S}$ 值为 $7.0 \%$, 夏季的 $\delta^{34} \mathrm{~S}$ 值为 $3.3 \%$, 而浙江中
部地区冬季和夏季的温差大约在 $15 \sim 20^{\circ} \mathrm{C}$, 因此可以 认定单一的因素还不能完全解释研究区域大气 $\mathrm{SO}_{2}$ 硫同位素的季节性变化, 同位素平衡分馏的温度效 应和夏季富轻同位素的生物成因硫的大量释放可能 是引起这种季节性变化的主要控制因素.

\section{3 大气 $\mathrm{SO}_{2}$ 氧化形成硫酸盐的 $\delta^{34} \mathrm{~S}$ 模式}

\subsection{1 $\mathrm{SO}_{2}$ 氧化途径及相关反应}

大气环境中 $\mathrm{SO}_{2}$ 转化形成硫酸盐气溶胶的机理 是酸沉降研究中人们关注的焦点问题. 实验研究已 经揭示了大量潜在的重要的氧化剂和氧化途径, 但 是定量描述大气环境中各种氧化机制仍然存在较大 的困难, 因此气相、云滴以及气溶胶微粒湿表面的 $\mathrm{SO}_{2}$ 氧化过程仍然还不清楚 ${ }^{[13]}$. 大气中的 $\mathrm{SO}_{2}$ 除部分 被植物、地面等直接吸收外，绝大部分被氧化形成硫 酸盐, 其中均相氧化和多相氧化是两种主要的氧化 
途径(图 5). 这些氧化反应途径的相对重要性是有争 论的, 而硫同位素比值可以用来定量评估这些氧化 途径在大气 $\mathrm{SO}_{2}$ 转化过程中的相对重要性 ${ }^{[13,15,32]}$.

$\mathrm{SO}_{2}$ 的均相氧化反应主要是 $\mathrm{SO}_{2}$ 被大气中的 $\mathrm{OH}$, $\mathrm{HO}_{2}$ 和 $\mathrm{RO}_{2}$ 自由基氧化. 在正常的大气条件下, $\mathrm{OH}$ 自 由基与 $\mathrm{HO}_{2}$ 自由基氧化速度之比大约为 12 , 也就是 说在 $\mathrm{SO}_{2}$ 的大气气相氧化中, $\mathrm{SO}_{2}$ 被 $\mathrm{OH}$ 自由基氧化 的反应占主要的比例 ${ }^{[33]}$, 其均相氧化反应可以用以 下反应式表示:

$$
\begin{aligned}
\mathrm{SO}_{2}+\mathrm{OH} & \rightarrow \mathrm{HOSO}_{2} \\
\mathrm{HOSO}_{2}+\mathrm{O}_{2} & \rightarrow \mathrm{SO}_{3}+\mathrm{HO}_{2} \\
\mathrm{SO}_{3}+\mathrm{H}_{2} \mathrm{O} & \rightarrow \mathrm{H}_{2} \mathrm{SO}_{4}
\end{aligned}
$$

在上面的反应中, 式(1) $\mathrm{HOSO}_{2}$ 自由基的形成是产生 硫同位素分馏的主要过程, 反应(2)和(3)由于反应速 率相当快, 硫同位素的分馏很小, 一般不予考虑.

$\mathrm{SO}_{2}$ 的多相氧化反应主要是 $\mathrm{SO}_{2}$ 溶于云、雾滴中, 被 $\mathrm{H}_{2} \mathrm{O}_{2}, \mathrm{O}_{2}$ 和 $\mathrm{O}_{3}$ 等液相氧化, 主要的多相氧化反应 可以用以下反应式表示:

$$
\begin{gathered}
\mathrm{SO}_{2}(\mathrm{~g}) \rightarrow \mathrm{SO}_{2}(\mathrm{aq}) \\
\mathrm{SO}_{2}(\mathrm{aq})+\mathrm{H}_{2} \mathrm{O} \rightarrow \mathrm{HSO}_{3}^{-}+\mathrm{H}^{+}
\end{gathered}
$$

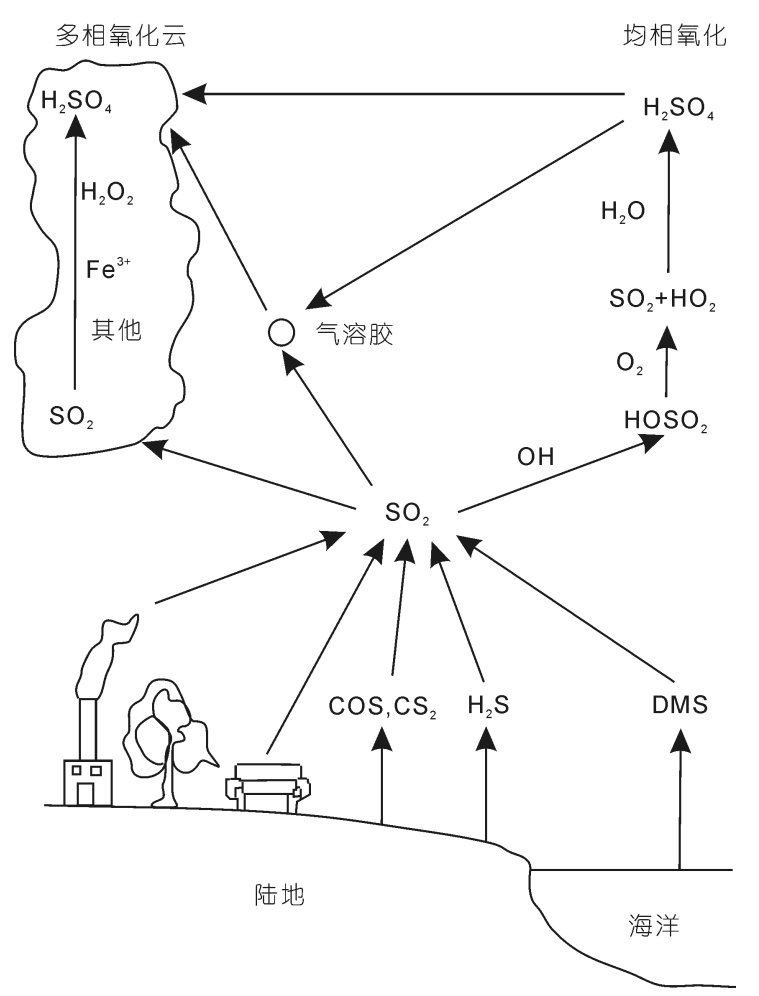

图 5 大气的 $\mathrm{SO}_{2}$ 来源和氧化途径

$$
\mathrm{HSO}_{3}{ }^{-}+\mathrm{H}_{2} \mathrm{O}_{2} \rightarrow \mathrm{SO}_{4}{ }^{2-}+\mathrm{H}^{+}+\mathrm{H}_{2} \mathrm{O}
$$

在 $\mathrm{SO}_{2}$ 的多相氧化反应中, $\mathrm{SO}_{2}$ 也被 $\mathrm{Fe}(\mathrm{III})$, $\mathrm{Mn}(\mathrm{IV}), \mathrm{O}_{3}$ 等氧化, 但是根据 $\mathrm{Martin}^{[34]}$ 的研究, 在 $\mathrm{pH}<4$ 的液相体系中 $\mathrm{H}_{2} \mathrm{O}_{2}$ 支配了 $\mathrm{SO}_{2}$ 的氧化反应. 浙 江中部地区大气降水的 $\mathrm{pH}$ 平均值约为 4.5 , 而云水的 酸度一般都要比降水要高, 因此下面的讨论不再涉 及其他氧化剂的反应. 另外. 云中四价硫的氧化反应 的速率要比大气中的气相氧化反应快, 模型计算表 明在全球范围内, 平流层云中四价硫的氧化反应速 度是云外氧化的 $2 \sim 5$ 倍 ${ }^{[35]}$.

\subsection{2 $\mathrm{SO}_{2}$ 氧化过程的 $\delta^{34} \mathrm{~S}$ 模式}

Eriksen ${ }^{[30,31]}$ 和 Egiazarov 等 ${ }^{[29]}$ 通过实验方法研究 了 $\mathrm{SO}_{2}-\mathrm{HSO}_{3}{ }^{-}$体系的硫同位素分馏系数, $25^{\circ} \mathrm{C}$ 反应式 (5)的分馏系数为 $1.0165 \pm 0.001$, 并且观察到反应产物 富集重硫同位素，同时反应的分馏系数随着温度降 低而升高. 反应式(3)和(6)的同位素分馏系数则要小 的多, 分馏系数的值大约为 $1.001^{[31]}$. Saltzman 等 ${ }^{[13]}$ 在上面工作的基础上, 提出利用 $\mathrm{SO}_{2}$ 氧化过程的同位 素分馏及大气 $\mathrm{SO}_{2}$ 和硫酸盐气溶胶的硫同位素数据 评估均相和多相氧化相对重要性的模式.

Saltzman 等 ${ }^{[13]}$ 用下面的公式来计算大气 $\mathrm{SO}_{2}$ 和 氧化形成的硫酸盐之间的同位素分馏:

$$
\alpha_{\mathrm{m}}=\frac{\frac{\delta^{34} \mathrm{SO}_{4}^{2-}}{10^{3}}+1}{\frac{\delta^{34} \mathrm{SO}_{2}}{10^{3}}+1} .
$$

由于 $\mathrm{SO}_{2}$ 或 $\mathrm{HSO}_{3}^{-}$在大气中的氧化反应是不可 逆的, 因此大气氧化反应中 $\mathrm{SO}_{2}$ 和硫酸盐是达不到同 位素平衡的. 硫酸盐形成过程中, 汇的效应可能造成 测定的同位素分馏 $\left(\alpha_{\mathrm{m}}\right)$ 与真实的同位素分馏并不一 致 ${ }^{[13]}$, 他们认为汇效应对 $\alpha_{\mathrm{m}}$ 的影响程度可以通过 Rayleigh 模型方程进行校正, 并得出真正的同位素分 馏系数的计算公式:

$$
\alpha_{\mathrm{c}}=\frac{\ln \frac{\delta^{34} S_{\mathrm{SO}_{2}}+10^{3}}{\delta^{34} \sum S+10^{3}}}{\ln f}+1,
$$

其中 $\delta^{34} \Sigma \mathrm{S}$ 是总大气硫的硫同位素, 包括 $\mathrm{SO}_{2}$ 和硫酸 盐，可以用下式表达:

$$
\delta^{34} \Sigma \mathrm{S}=\frac{\left[\mathrm{SO}_{2}\right] \times \delta^{34} \mathrm{SO}_{2}+\left[\mathrm{SO}_{4}^{2-}\right] \times \delta^{34} \mathrm{SO}_{4}^{2-}}{\left[\mathrm{SO}_{2}\right]+\left[\mathrm{SO}_{4}^{2-}\right]} .
$$

浙江中部地区大气中 $\mathrm{SO}_{2}$ 氧化形成硫酸盐的过 
程中存在着一定的同位素效应, 这可以从表 1 的数据 和图 2 的比较中得出结论, 硫酸盐气溶胶的 $\delta^{34} \mathrm{~S}$ 均值 比大气 $\mathrm{SO}_{2}$ 高 $3.4 \%$, 大气 $\mathrm{SO}_{2}$ 的氧化产物硫酸盐更 富轻硫同位素. 大气环境 $\mathrm{SO}_{2}$ 的氧化过程主要有两种 不同同位素分馏机制, 一种是 $\mathrm{SO}_{2}$ 溶解(反应式(5))伴 随的平衡分馏, 重硫同位素积聚在液滴中, 而残留气 相的硫富集轻同位素. 另一种就是氧化反应过程伴 随的动力学同位素效应, 由于 ${ }^{32} \mathrm{~S}$ 的反应速度比 ${ }^{34} \mathrm{~S}$ 快, 导致反应产物富集轻同位素 $(\alpha=0.996)$. Saltzman 等 ${ }^{[13]}$ 认为不同氧化剂和反应机理, 动力学分馏的程 度是变化的, 但总体上动力学同位素效应要大大小 于平衡分馏. $\mathrm{SO}_{2}$ 均相氧化过程仅存在动力学分馏, 其氧化反应必然导致反应产物硫酸盐富集轻硫同位 素, 而 $\mathrm{SO}_{2}$ 多相氧化过程则包含动力学分馏和平衡分 馏两种硫同位素分馏机制, 氧化反应的产物硫酸盐 则会比 $\mathrm{SO}_{2}$ 富集重硫同位素 $(\alpha>1)$. 因此, 从我们的 分析数据可以得出这样的结论, 浙江中部地区大气 $\mathrm{SO}_{2}$ 氧化形成硫酸盐的过程中多相氧化可能起了主 导作用. 表 2 是按照大气 $\mathrm{SO}_{2}$ 和硫酸盐气溶胶 $\delta^{34} \mathrm{~S}$ 值 测定数据计算的同位素分馏系数 $\alpha_{\mathrm{m}}$, 由于我们没有 测定气溶胶中硫酸盐的浓度 $\left[\mathrm{SO}_{4}{ }^{2-}\right]$, 无法直接计算 校正后的同位素分馏系数 $\alpha_{\mathrm{c}}$, 但 Saltzman 等 ${ }^{[13]}$ 在 Hubbard Brook 的计算结果表明, 汇效应引起的影响 程度约在 0.001 或者更小, 因此我们可以通过 $\alpha_{\mathrm{m}}$ 大致 估计均相氧化和多相氧化这两种氧化机制的相对重 要性. $\alpha_{\mathrm{m}}$ 数据在 $1.000 \sim 1.009$ 之间, 同样表明与均相 氧化比较, 大气 $\mathrm{SO}_{2}$ 的多相氧化应该处于主导作用, 但相对适中的 $\alpha_{\mathrm{m}}$ 数据同时也表明均相氧化在浙江中 部地区的氧化反应机制中起着不可低估的作用.

表 2 计算的大气 $\mathrm{SO}_{2}$ 氧化反应同位素分馏系数 ${ }^{\mathrm{a})}$

\begin{tabular}{ccc}
\hline 时间 & $\Delta \delta^{34} \mathrm{~S}$ & $\alpha_{\mathrm{m}}$ \\
\hline 2005 年 1 月 & 1.3 & 1.0013 \\
2005 年 2 月 & 0.2 & 1.0003 \\
2005 年 3 月 & 1.5 & 1.0015 \\
2005 年 4 月 & 1.4 & 1.0014 \\
2005 年 5 月 & 8.8 & 1.0089 \\
2005 年 6 月 & 4.6 & 1.0047 \\
2005 年 7 月 & 5.3 & 1.0054 \\
2005 年 8 月 & 3.5 & 1.0035 \\
2005 年 9 月 & 6.2 & 1.0061 \\
2005 年 10 月 & 3.2 & 1.0031 \\
2005 年 11 月 & 2.7 & 1.0027 \\
2005 年 12 月 & 1.4 & 1.0014 \\
\hline
\end{tabular}

a) $\Delta \delta^{34} \mathrm{~S}$ 为气溶胶和大气 $\mathrm{SO}_{2}$ 硫同位素比值的差值
浙江中部地区的大气 $\mathrm{SO}_{2}$ 的氧化机制基本与欧 洲中部的捷克相似, 都以多相氧化占主导 ${ }^{[15]}$, 但浙江 中部地区均相氧化所起的作用要稍大一些. 从理论 上讲, 由于温度的增加 $\mathrm{SO}_{2}$ 在水中的溶解度会减小, 并且大气中氧化剂的光化学产生量在夏季达到最 $大^{[13]}$, 因此夏季均相氧化在大气 $\mathrm{SO}_{2}$ 的氧化反应中 起着相对重要的作用, 而冬天多相氧化所起的作用 更大. 浙江中部地区大气 $\mathrm{SO}_{2}$ 的氧化过程中并没有体 现这种季节性的氧化机制, Mukai 等 ${ }^{[12]}$ 在中国的北方 地区观察到了相应的氧化反应特征, 但在南方的贵 阳夏季仍然表现出相对较高的分馏系数, 这和浙江 中部地区观察到的结果是一致的. 有两个因素可以 解释这种与理论并不完全一致的现象, 一个是国内 城市的大气污染相对比较严重, 大气含有较高浓度 的气溶胶; 另一个原因就是南方气候湿润, 春、夏季 大气的相对湿度很高, 这两个因素增加了大气中水 滴和湿气溶胶的微粒表面, 使大气 $\mathrm{SO}_{2}$ 优先与氧化剂 发生多相氧化反应. 图 6 是我们发现的一个有趣的现 象, 它与上面的分析结论非常吻合, 从图中可以看到 大气环境的相对湿度与 $\mathrm{SO}_{2}$ 氧化形成硫酸盐过程的 硫同位素分馏系数 $\alpha$ 存在着比较明显的依赖关系, 春 季 5 月份大气相对湿度最大时, 大气 $\mathrm{SO}_{2}$ 氧化反应的 同位素分馏系数也达到最大, 表明相对湿度对大气 $\mathrm{SO}_{2}$ 的氧化机制有着重要的影响.

\section{3 结论}

(1) 浙江中部地区环境空气中 $\mathrm{SO}_{2}$ 日均值浓度在 $0.004 \sim 0.204 \mathrm{mg} / \mathrm{m}^{3}$ 之间, 年平均值为 $0.046 \mathrm{mg} / \mathrm{m}^{3}$; $\mathrm{NO}_{2}$ 日均值浓度在 $0.010 \sim 0.135 \mathrm{mg} / \mathrm{m}^{3}$ 之间, 年平均 值为 $0.055 \mathrm{mg} / \mathrm{m}^{3}$. 大气 $\mathrm{SO}_{2}$ 和 $\mathrm{NO}_{2}$ 都表现出比较明 显的季节性变化, $\mathrm{SO}_{2}$ 浓度冬、春季比较高, 夏、秋季 相对较低.

(2) 浙江中部地区大气 $\mathrm{SO}_{2}$ 的 $\delta^{34} \mathrm{~S}$ 值变化范围在 $1.0 \% 0 \sim 7.5 \%$ 之间, 年均值为 $4.7 \% \circ \pm 2.3 \%$, 气溶胶 $\delta^{34} \mathrm{~S}$ 值变化范围在 $6.4 \%$ $9.8 \%$ 之间, 年均值为 $8.1 \%$ 王 $1.0 \%$. 气溶胶 $\delta^{34} \mathrm{~S}$ 值均大于相应的大气 $\mathrm{SO}_{2}$ 的 $\delta^{34} \mathrm{~S}$ 值, 两者的年均值相差 $3.4 \%$.

(3) 大气 $\mathrm{SO}_{2}$ 的 $\delta^{34} \mathrm{~S}$ 值季节性变化明显, 冬季到 春季的 4 月份 $\delta^{34} \mathrm{~S}$ 值最高, 春末到夏季的 $\delta^{34} \mathrm{~S}$ 值最低, 秋季比夏季稍高. 气溶胶 $\delta^{34} \mathrm{~S}$ 值全年的变化幅度不大, 基本围绕在年均值附近上下波动, 春季的 $\delta^{34} \mathrm{~S}$ 值相对 


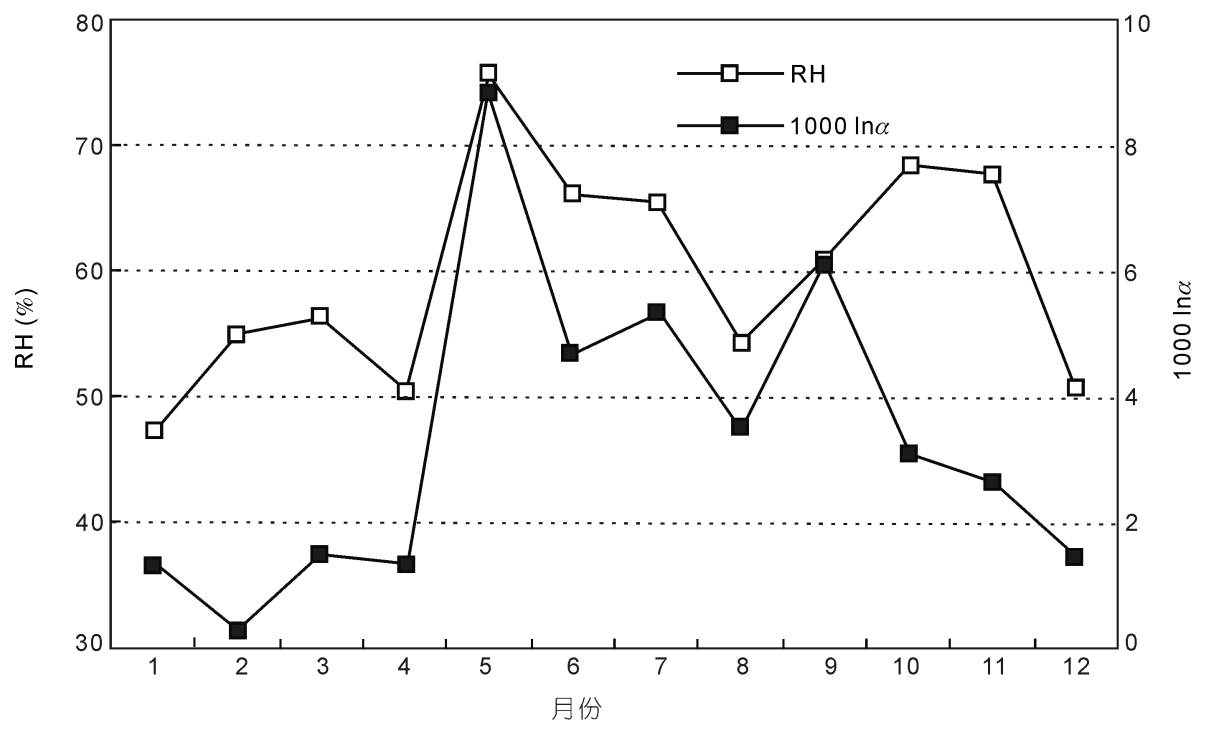

图 $6 \mathrm{SO}_{2}$ 氧化过程硫同位素分馏系数与相对湿度的相互变化

稍高, 冬、夏和秋季的 $\delta^{34} \mathrm{~S}$ 值几乎完全一致.

(4) 浙江中部地区大气 $\mathrm{SO}_{2}$ 冬季的 $\delta^{34} \mathrm{~S}$ 值为 $7.0 \%$, 夏季的 $\delta^{34} \mathrm{~S}$ 值为 $3.3 \%$, 研究认为单一的因素 还不能完全解释研究区域大气 $\mathrm{SO}_{2}$ 硫同位素的季节 性变化, 同位素分馏平衡的温度效应和夏季富轻同 位素的生物成因硫的大量释放可能是引起这种季节
性变化的主要控制因素.

(5) 本文讨论了大气 $\mathrm{SO}_{2}$ 氧化形成硫酸盐的 $\delta^{34} \mathrm{~S}$ 模式, 认为在浙江中部地区大气 $\mathrm{SO}_{2}$ 氧化反应中多相 氧化处于主导地位，同时均相氧化在氧化反应机制 中也有其不可低估的作用. 同时, 研究区域大气环境 的相对湿度对大气 $\mathrm{SO}_{2}$ 的氧化机制有着重要的影响.

\section{参考文献}

1 Jamieson R E, Wadleigh M A. Tracing sources of precipitation sulfate in eastern Canada using stable isotopes and trace metals. J Geophy Res, 2000, 105: 20549-20556

2 Romero A B. Mass-independent sulfur isotopic compositions in atmospheric sulfates. Doctoral Dissertation. San Diego: University of California, 2003

3 Bao H M, Reheis M C. Multiple oxygen and sulfur isotopic analyses on water-soluble sulfate in bulk atmospheric deposition from the southwestern United States. J Geophy Res, 2003, 108: 4430-4438

4 Norman A L, Belzer W, Barrie L A. Insights into the biogenic contribution to total sulphate in aerosol and precipitation in the Fraser Valley afforded by isotopes of sulphur and oxygen. J Geophy Res, 2004, 109: 5311-5320

5 Tie X, Brasseur G P, Briegleb B, et al. Two-dimensional simulation of Pinatubo aerosol and its effect on stratospheric ozone. J Geophy Res, 1994, 99: 20545-20562

6 Adams P J, Seinfeld J H, Koch D M. Global concentrations of tropospheric sulfate, nitrate, and ammonium aerosol simulated in a general circulation model. J Geophy Res, 1999, 104: 13791-13823

7 Charlson R J, Schwartz S E, Hales J M, et al. Climate forcing by atmospheric aerosols. Science, 1992, 225: 423-430

8 Seinfeld, J H, Pandis S N. Atmospheric Chemistry and Physics: From Air Pollution to Climate Change. New York: John Wiley \& Sons, Inc, 1998

9 Haywood J, Boucher O. Estimates of the direct and indirect radiative forcing due to tropospheric aerosols: A review. Rev Geophy, 2000, 38: 513-543

10 IPCC. Climate change 2001: The scientific basis. In: Contribution of Working Group I to the Third Assessment Report of the Inter- 
government Panel on Climate Change. New York: Cambridge University Press, 2001

11 张苗云. 酸沉降环境化学与硫同位素示踪研究——以浙江中部地区为例. 博士学位论文. 北京: 中国科学院研究生院, 2006. 1-136

12 Mukai H, Tanaka A, Fujii T, et al. Regional characteristics of sulfur and lead isotope ratios in the atmosphere at several Chinese urban sites. Environ Sci Technol, 2001, 35: 1064-1071

13 Saltzman E, Brass G, Price D. The mechanism of sulphate aerosol formation: Chemical and sulphur isotopic evidence. Geophy Res Lett, 1983, 10: 513-516

14 Kawamura H, Matsuoka N, Tawaki S, et al. Sulfur isotope variations in atmospheric sulfur oxides, particulate matter and deposits collected at Kyushu Island, Japan. Water Air Soil Pollut, 2001, 130: 1775-1780

15 Novak M, Jackova I, Prechova E. Temporal trends in the isotope signature of air-borne sulfur in Central Europe. Environ Sci Technol, 2001, 35: $255-260$

16 张鸿斌, 陈毓蔚, 刘德平. 广州地区酸雨硫源的硫同位素示踪研究. 地球化学, 1995, 24(增刊): 126-133

17 张鸿斌, 胡露琴, 卢承祖. 华南地区酸沉降的硫同位素组成及其环境意义. 中国环境科学, 2002, 22: 165-169

18 洪业汤, 张鸿斌, 朱泳煊, 等. 中国煤的硫同位素组成特征及燃煤过程的硫同位素分馏. 中国科学 B 辑, 1992, 22: 868-873

19 洪业汤, 朱泳煊, 张鸿斌, 等. 燃煤过程硫同位素分馏效应及其环境意义. 环境科学学报, 1993, 13: 240-243

20 洪业汤, 张鸿斌, 朱泳煊, 等. 西南酸雨来源的稳定硫同位素研究. 贵阳: 中国科学院地球化学研究所, 1990

21 刘广深，洪业汤，朴河春，等. 用稳定同位素方法探讨大气颗粒物中硫的来源. 中国环境科学, 1996, 16: 426-429

22 Norman A L, Barrie, L A, Toom-Sauntry D, et al. Sources of aerosol sulphate at Alert: Apportionment using stable isotopes. J Geophy Res, 1999, 104: 11619-11631

23 Nriagu J O, Coker R D. Isotopic composition of sulphur in precipitation within the Great Lakes basin. Tellus, 1978, 30: 365-375

24 Nriagu J O, Holdway D A, Coker R D. Biogenic sulphur and the acidity of rainfall in remote areas of Canada. Science, 1987, 237: $1189-1192$

25 van Stempvoort D R, Wills J J, Fritz P. Aboveground vegetation effects on the deposition and cycling of atmospheric sulfur: Chemical and stable isotope evidence. Water Air Soil Pollut, 1991, 60: 55-82

26 洪业汤, 张鸿斌, 朱泳煊, 等. 中国大气降水的硫同位素组成特征. 自然科学进展一一国家重点实验室通讯, 1994, 4: 741-745

27 Caron F, Tessier A, Kramer J R, et al. Sulphur and oxygen isotopes of sulphate in precipitation and lake water, Quebec. Appl Geochem, 1986, 1: 601-606

28 Alewell C, Mitchell M J, Likens, G E, et al. Assessing the origin of sulfate deposition at the Hubbard Brook Experimental Forest. J Environ Qual, 2000, 29: 759-767

29 Egiazarov A C, Kaviladze M N, Kerner E L, et al. Separation of sulfur isotopes by chemical exchange. Isotopenpraxis, 1971, 7: 379-383

30 Eriksen T E. Sulfur isotope effects, I. The isotopic exchange coefficient for the sulfur isotopes ${ }^{34} \mathrm{~S}-{ }^{32} \mathrm{~S}$ in the system $\mathrm{SO}_{2 \mathrm{~g}}-\mathrm{HSO}_{3-\mathrm{ag}}$ at 25,35 and $45^{\circ} \mathrm{C}$. Acta Chem Scand, 1972, 26: 573-580

31 Eriksen T E. Sulfur isotope effects, II. The isotopic exchange coefficients for the sulfur isotopes ${ }^{34} \mathrm{~S}^{32} \mathrm{~S}$ in the system $\mathrm{SO}_{2} \mathrm{~g}$-aqueous solutions of $\mathrm{SO}_{2}$. Acta Chem Scand, 1972, 26: 581-584

32 Tanaka N, Rye D M, Xiao Y T, et al. Use of stable sulfur isotope systematics for evaluating oxidation reaction pathways and in-cloudscavenging of sulfur dioxide in the atmosphere. Geophy Res Lett, 1994, 21: 1519-1522

33 唐孝炎. 大气环境化学. 北京: 高等教育出版社, 1992

34 Martin L R. Kinetics studies of sulfite oxidation in aqueous solution, in $\mathrm{SO}_{2}, \mathrm{NO}$ and $\mathrm{NO}_{2}$ oxidation mechanisms: atmospheric consideration. In: Teasley J I, ed. Acid Precipitation Series. Boston: Butterworth Publishers, 1984. 66-100

35 Langner J, Rodhe H. A global three-dimensional model of the tropospheric sulfur cycle. J Atmos Chem, 1991, 13: 255-263 